\title{
$\underline{\mathbf{P}-146}$
}

\section{Centrifugal Partition Chromatography (CPC) as an Effective Method for Natural Products Purification}

\author{
Chan Gomathi* \\ Application Consultant, Orbiting Scientific and Technology Sdn. Bhd., 35-1, Jalan Radin Anum 1, Seri Petaling, 57000 \\ Kuala Lumpur, Malaysia; E-mail: changomathi@orbitingscientific.com
}

\begin{abstract}
Natural products purification has often been a challenging task to many scientists worldwide. Many techniques have been invented and upgraded to make this task easier. This presentation will depict a few examples of natural products purification by using Centrifugal Partition Chromatography (CPC), which demonstrates a fast and effective way of natural products isolation. One example is CPC was applied to fraction F1 (hexane-ethyl acetate, 95:5) of hexane extract from Mesua elegans (Clusiaceae). Two 4-phenylcoumarins; mammea A/BA and mesuagenin $\mathrm{C}$, was obtained from the fraction, with purity of $93.2 \%$ and $94.3 \%$ in $500 \mathrm{mg}$ injection in multi-dual mode compare to $82.3 \%$ and $79.4 \%$ in isocratic mode. Another example is the development of a versatile tool for fast screening and rapid detection of bioactive natural products from plant extracts: the on-line coupling of CPC-UV to HPLC-UV-MS, via a six position switching valve. This strategy offers the possibility to get instant HPLC fingerprint of fractions and structural information about separated molecules during the CPC fractionation step. This new approach was applied to the fractionation and purification of xanthones from Garcinia mangostana (Clusiaceae) pericarp. The combined CPC-HPLC-DAD-MS allows the simultaneous fractionation, detection and characterization of sixteen molecules. Ten molecules were identified based on their UV and MS spectra. Furthermore, this method has led to the isolation of $\alpha$-mangostin and $\gamma$-mangostin at $98.0 \%$ and $98.5 \%$ purity in a very short time.
\end{abstract}

Keywords: Centrifugal Partition Chromatography (CPC), natural products. 